\title{
Perceived Size and Spatial Coding
}

\author{
Derek H. Arnold, Annette Birt, and Thomas S. A. Wallis \\ School of Psychology, The University of Queensland, St. Lucia, Queensland 4072, Australia
}

Images of the same physical dimensions on the retina can appear to represent different-sized objects. One reason for this is that the human visual system can take viewing distance into account when judging apparent size. Sequentially presented images can also prompt spatial coding interactions. Here we show, using a spatial coding phenomenon (the tilt aftereffect) in tandem with viewing distance cues, that the tuning of such interactions is not simply determined by the physical dimensions of retinal input. Rather, we find that they are contingent on apparent size. Our data therefore reveal that spatial coding interactions in human vision are modulated by processes involved in the determination of apparent size.

Key words: spatial coding; perceived size; retinotopy; tilt aftereffect; size distance invariance; perception

\section{Introduction}

The tilt aftereffect is perhaps one of the best known spatial illusions. It can occur after prolonged exposure to an oriented adapting stimulus. Afterward, stimuli presented at the same position as the adaptor can appear rotated away from the adapted orientation (Gibson and Radner, 1947).

Although the neurophysiological substrate/s of the tilt aftereffect are not yet comprehensively understood, it is thought to be causally related to interactions between orientation-selective mechanisms in the primary, and/or extrastriate, visual cortex (Blakemore et al., 1970; Coltheart, 1971; Clifford et al., 2000). A cortical locus is probable because subcortical neurons do not show strong orientation tuning (Smith et al., 1990; Xu et al., 2002). Also, the illusion magnitude has been found to correlate with blood oxygenation level-dependent signal changes in human primary visual cortex (Fang et al., 2005; Liu et al., 2007).

The research question to be addressed here is whether the position-based tuning of the tilt aftereffect reflects the physical or apparent spatial overlap between adapting and subsequent test stimuli. We can ask this question because the size of an object's image on the retina does not predict its apparent size. For instance, movement can be used to induce a size illusion, because apparent position can be shifted toward the direction of motion (De Valois and De Valois, 1991; Whitney et al., 2003). Alternatively, viewing distance cues can be used to distort apparent size via size-depth invariance, meaning that there is an approximately constant ratio between the apparent size of an object and its apparent distance in depth from the observer (Emmert, 1881; Holway and Boring, 1941; Joynson, 1949; Ono, 1966). Because of this, people do not typically seem to shrink as they walk away from us or to grow as they move toward us, despite the fact that the retinal images of people in these cases do shrink and expand.

Received 0ct. 15, 2007; revised April 9, 2008; accepted April 12, 2008.

This work was supported by a Discovery Project Grant and Australian Postdoctoral Fellowship awarded to D.H.A. by the Australian Research Council. We are grateful to Jason Mattingley, Phillip Grove, and Signy Wegener.

Correspondence should be addressed to Derek H. Arnold at the above address. E-mail: darnold@psy.uq.edu.au. DOI:10.1523/JNEUROSCI.0578-08.2008

Copyright @ 2008 Society for Neuroscience $\quad$ 0270-6474/08/285954-05\$15.00/0
To determine whether the tuning of the tilt aftereffect is contingent on perceived or physical size, we will first determine the magnitude of a size illusion induced using distance cues that are extrinsic to the adapting and test stimuli (experiment 1). We will then determine whether the tuning of the tilt aftereffect is better predicted by the physical or apparent spatial overlap of the adapting and test stimuli (experiment 2).

\section{Materials and Methods}

General. There were eight observers, the three authors and five volunteers who were naive as to the purpose of the experiments. All had normal or corrected-to-normal vision.

Visual stimuli were generated using Matlab software in conjunction with the Psychophysics toolbox (Brainard, 1997; Pelli, 1997). They were presented on two Samsung Syncmaster 750 s monitors $(1024 \times 768$ resolution, $75 \mathrm{~Hz}$ refresh rate) located to the left and right of the observer and viewed via half-silvered front surface mirrors (Fig. 1a,b).

One of the two monitors was located at an optical distance of $45 \mathrm{~cm}$ from the observer, whereas the other was $90 \mathrm{~cm}$ from the observer. Two red diodes served as fixation points. These were positioned $45 \mathrm{~cm}$ (near fixation point) and $90 \mathrm{~cm}$ (far fixation point) in front of the observer and could be seen through the half-silvered mirrors (Fig. 1a,b). Only one of the two diodes was lit at a time $\left(8.5 \mathrm{~cd} / \mathrm{m}^{2}\right)$. The vergence angle of the eyes, when fixated on either lit diode, provided the visual system with a salient depth cue.

Before each run of trials, observers completed a simple calibration task by which we determined coordinates, on each monitor, that corresponded with the near and far fixation points. This involved positioning sets of crosshairs, sequentially on each monitor, until they seemed to target the lit diode.

A gray visual frame was constructed to provide further depth cues (Fig. $1 c, d$ ). This consisted of a binocular square outline (width $=35.4^{\circ}$; line widths $=0.6^{\circ}$ ) centered on monitor coordinates that corresponded with the near fixation point. There was therefore no horizontal binocular disparity between the two eye views of this square when observers fixated the near fixation point. However, when observers fixated the far fixation point, there was a crossed horizontal binocular disparity signal consistent with a viewing distance difference of $45 \mathrm{~cm}$.

Four lines (widths $=0.6^{\circ}$ ) extended from the corners of the square outline and terminated at coordinates corresponding with the corners of an invisible square (width $=17.7^{\circ}$ ) centered on the far fixation point (Fig. 1c,d). When observers fixated the far fixation point, there was no 

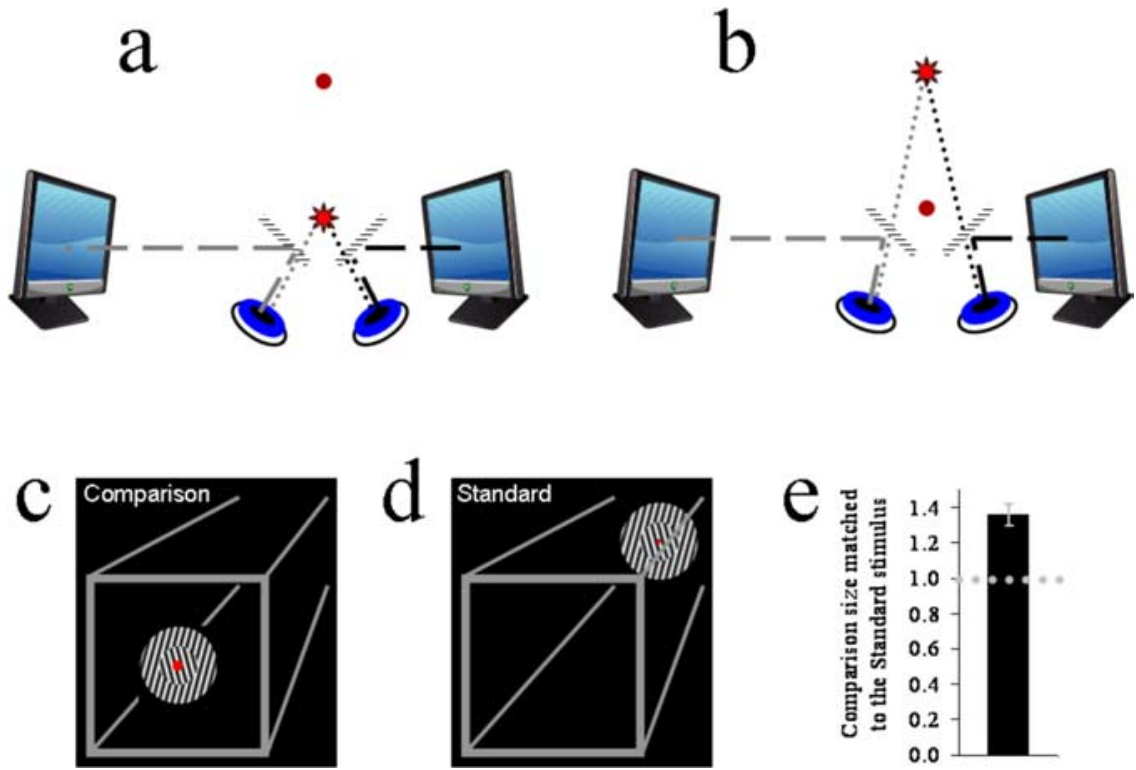

Figure 1. $\quad \boldsymbol{a}, \boldsymbol{b}$, Graphics depicting the apparatus used in these experiments. $\boldsymbol{c}, \boldsymbol{d}$, Graphics depicting the three-dimensional visual frame used in all experiments, and the subjective appearance of the standard and comparison stimuli in experiment 1.e Bar plot showing the comparison stimulus size matched to the standard stimulus as a proportion of the standard stimulus size. The dotted horizontal line shows a proportion of 1, at which there would be no size illusion. The error bar shows SEM.

binocular disparity associated with the images of the far ends of these lines. However, when observers fixated on the near fixation point, there was an uncrossed horizontal binocular disparity signal consistent with a viewing distance difference of $45 \mathrm{~cm}$.

All test and adapting stimuli (Figs. $1 c, d, 2 a, b)$ were shown on both monitors, and the two images of the stimuli were physically scaled so that they matched in terms of retinal image size and spatial frequency. The two images of stimuli were also always centered on monitor coordinates corresponding with the current fixation point. There was therefore never any binocular disparity associated with them. Thus all test and adapting stimuli appeared to be located at the same distances from the observers as the current fixation point: either 45 or $90 \mathrm{~cm}$ in front of the observer.

Readers should note that the depth cues used in these experiments were extrinsic to the test and adapting stimuli, being given by the vergence angle of the eyes and by the surrounding frame (Fig. $1 a-d$ ). There were therefore no physical differences between the retinal images of test and adapting stimuli across the critical experimental conditions.

Experiment 1: determining the magnitude of a size illusion. We first determined the magnitude of a size illusion induced using a combination of two depth cues, the vergence angle of the eyes (Fig. 1a,b) and binocular disparity signals within a visual frame that surrounded the test stimuli (Fig. 1c,d).

The standard stimulus consisted of a circle with an inner and an outer sector, each containing a differently oriented grating (Fig. $1 d$ ). The entire circle subtended $11^{\circ}$ of visual angle, whereas the inner sector subtended $5.5^{\circ}$. The outer grating was oriented either at $-15^{\circ}$ from vertical (and the inner at $+15^{\circ}$ ) or at $+15^{\circ}$ from vertical (and the inner at $-15^{\circ}$ ). These combinations were determined at random on a trial-by-trial basis. The phase of the grating waveforms was set to either 0 or $180^{\circ}$, again determined at random on a trial-by-trial basis. The spatial frequency of the gratings within the standard stimulus was 2 cycles per degree of visual angle. The Michelson luminance contrast of the gratings was $100 \%$. Crucially, the standard stimulus was always presented in conjunction with the far fixation point, and thus it seemed to be located $90 \mathrm{~cm}$ in front of the observer (Fig. 1d)

The comparison stimulus was identical to the standard in all features with the following exceptions. First, on a trial-by-trial basis, the spatial attributes of the comparison were scaled ( $80-180 \%$, in step sizes of $10 \%)$ relative to those of the standard stimulus according to the method of constant stimuli. Second, the comparison was always presented in con- junction with the near fixation point. Thus, it appeared to be located $45 \mathrm{~cm}$ in front of the observer (Fig. 1c).

Each trial started with one of the two diodes being lit. There was a $1 \mathrm{~s}$ pause before either the standard (if the far fixation was lit) or a comparison (if the near fixation point was lit) was presented for $250 \mathrm{~ms}$. The initial pause provided ample time for the observer to fixate the lit diode before the stimulus was presented. After the first presentation, the first diode was turned off and the other fixation point was lit, followed by another $500 \mathrm{~ms}$ pause so that the observer could shift his or her point of fixation. Then, either a comparison (if the near fixation point was lit) or the standard (if the far fixation point was lit) stimulus was presented for $250 \mathrm{~ms}$. Both screens then went blank, and the observer indicated whether the second stimulus had appeared to be bigger or smaller than the first by pressing one of two buttons. The order in which stimuli were presented was determined at random on a trialby-trial basis.

Each observer completed two runs of trials, each consisting of 176 individual trials. These provided distributions of apparent comparison size (relative to the standard stimulus) as a function of physical size. Weibull functions were fitted to these data and $50 \%$ points taken as estimates of the physical comparison size perceptually matched to the standard stimulus. In Figure $1 e$, the average of these estimates is expressed as a proportion, such that values $>1$ indicate that physically larger comparison sizes were matched to the physically smaller standard stimulus.

Experiment 2: tilt aftereffect tuning as a function of apparent size. In experiment 2 , we determined whether the size illusion measured in experiment 1 would modulate the tuning of the tilt aftereffect.

The adapting stimulus was identical to the standard in experiment 1 with the following exceptions. First, during a run of trials, the outer grating of the adapting stimulus was always orientated $-15^{\circ}$ from verti$\mathrm{cal}$ (and the inner $+15^{\circ}$ ) or at $+15^{\circ}$ from vertical (and the inner $-15^{\circ}$ ). Second, to minimize the effects of localized contrast adaptation and perceptual fading, during adaptation periods the phase of the waveforms within the gratings alternated between 0 and $180^{\circ}$ at a rate of $1 \mathrm{~Hz}$.

During different runs of trials, adapting stimuli were presented in conjunction with (and centered on monitor coordinates corresponding to) the far or near fixation point. This created a strong impression that the adapting stimulus was located at 90 or $45 \mathrm{~cm}$, respectively, from the observer (Fig. 2a).

Test stimuli consisted of gratings presented within annuli (Fig. $2 b$ ). The spatial frequencies and luminance contrasts of gratings within test stimuli were identical to those within the adapting stimulus.

There were three types of test stimulus (Fig. 2b,c): (1) Inner tests. These overlapped exclusively with the inner sectors of adapting stimuli. They had an outer diameter subtending $5.53^{\circ}$ of visual angle and an inner diameter subtending $2.68^{\circ}$. (2) Central tests. These overlapped equally with both the inner and outer sectors of adapting stimuli. They had an outer diameter subtending $6.95^{\circ}$ of visual angle and an inner subtending $4.11^{\circ}$. (3) Outer tests. These overlapped exclusively with the outer sectors of adapting stimuli. They had an outer diameter subtending $8.37^{\circ}$ of visual angle and an inner diameter subtending $5.53^{\circ}$.

During different runs of the trials, test stimuli were presented in conjunction with either the near or the far fixation point. They therefore appeared to be located either 45 or $90 \mathrm{~cm}$, respectively, in front of the observer (Fig. $2 b$ ).

Each trial started with either the far or near fixation point being lit, depending on whether the adapting stimulus was coupled with the far or near fixation point. Then there was a $500 \mathrm{~ms}$ pause, which allowed the observer to fixate the lit diode. Exposure to the adapting stimulus then 

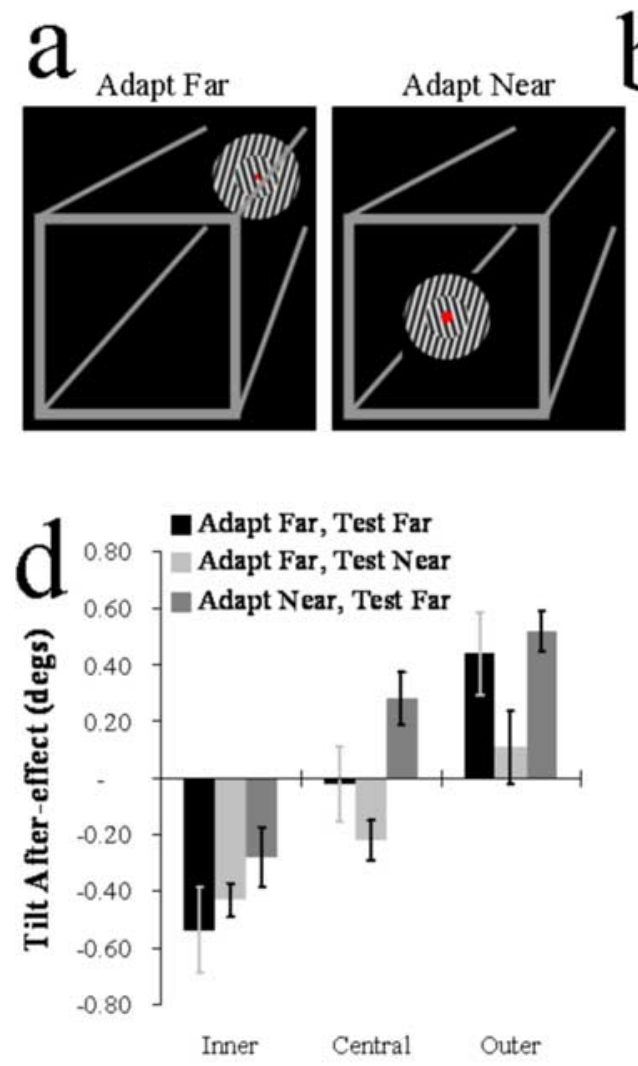

Test Eccentricity
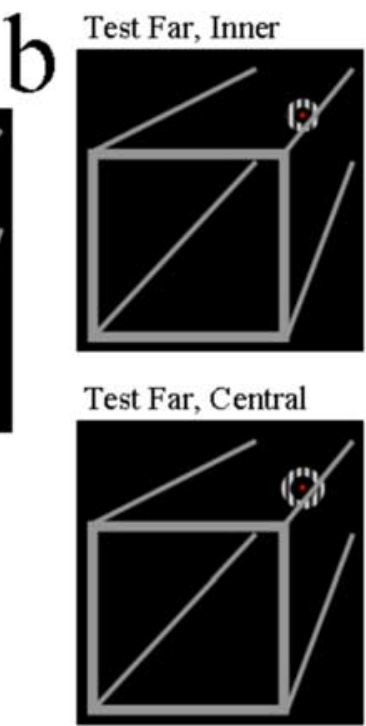

Test Far, Outer

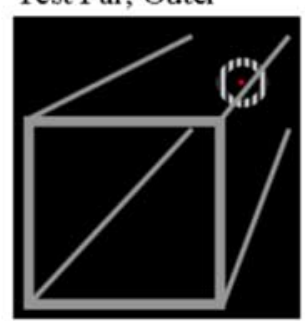

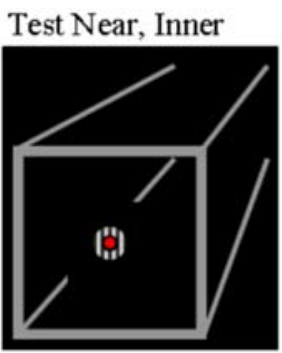

Test Near, Central

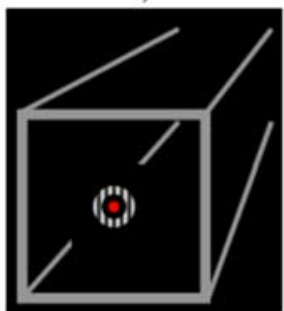

Test Near, Outer

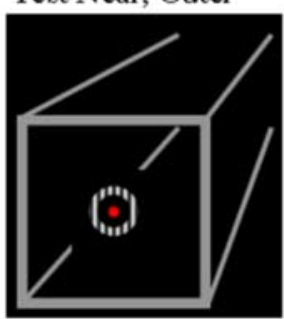

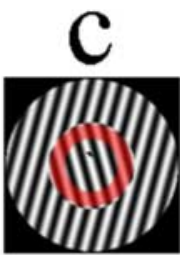

Inner Test

physical

overlap

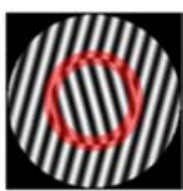

Central Test

physical

overlap

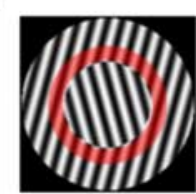

Outer Test

physical

overlap

Figure 2. $\quad \boldsymbol{a}$, Graphic depicting the subjective appearance of adapting stimuli in experiment 2. $\boldsymbol{b}$, Graphics depicting test stimuli from experiment 2. c, Graphics depicting the physical overlap between test and adapting stimuli. Regions of overlap are depicted by transparent red rings superimposed on depictions of adapting stimuli. $\boldsymbol{d}$, Bar plots showing the signed magnitude of tilt aftereffects. Negative values signify that test stimuli appeared rotated away from the orientations of gratings within inner sectors of adapting stimuli, and positive values signify that test stimuli appeared rotated away from the orientations of gratings within outer sectors. Error bars show SEM.

commenced, persisting for either $30 \mathrm{~s}$ (first trial) or $5 \mathrm{~s}$ (subsequent trials). The adapting stimulus then disappeared. Then either the far fixation point (test-far runs of trials) or near fixation point (test-near runs of trials) was lit. There was then another $500 \mathrm{~ms}$ pause, allowing observers to shift their point of fixation if required. A test stimulus was then presented for $250 \mathrm{~ms}$. The observer then indicated if the test grating had appeared to be tilted to the left or right of vertical by pressing one of two buttons.

During a run of trials, test stimulus orientations were manipulated, on a trial-by-trial basis $\left( \pm 3^{\circ}\right.$ from vertical), according to the method of constant stimuli. During a run of trials, each type of test stimulus was each presented in 56 trials in a random order. A complete run of trials therefore consisted of 168 individual trials. Each run of trials provided three distributions of apparent test stimulus orientation as a function of the physical test stimulus orientations. Weibull functions were fitted to these and $50 \%$ points taken as estimates of subjective vertical.

Each observer completed two test-far runs of trials and two test-near runs of trials. In both cases, during one of the two runs of trials the outer grating within the adapting stimulus was oriented $+15^{\circ}$ from vertical (and the inner at $-15^{\circ}$ ). These are referred to as outer tilted right runs of trials. During the other run of trials, the outer grating was oriented $-15^{\circ}$ from vertical (and the inner grating $+15^{\circ}$; outer tilted left runs of trials). The order in which these were completed was counterbalanced across observers.

Tilt aftereffects were calculated for each condition by taking one-half the difference between subjective vertical estimates from outer tilted left runs of trials and outer tilted right runs of trials. Doing so provides a signed value. Negative values show that test stimuli appeared rotated away from the orientations of gratings within inner sectors of adapting stimuli. Positive values show that test stimuli appeared rotated away from the orientations of gratings within outer sectors. A value of zero would signify that apparent test orientations were not influenced by preexposures to adapting stimuli.

\section{Results}

In experiment 1 , we found that comparison stimuli had to be $\sim 1.36$ times the physical size of the standard stimulus if the two were to seem to match. Our manipulation therefore induced a $36 \%$ size illusion $\left(t_{(7)}=5.4, p=0.001\right)$ with apparently closer stimuli reduced in size relative to physically identical stimuli that appeared to be more distant.

If the tuning of the tilt aftereffect were determined solely by the relative physical dimensions of retinal input, we should observe opposite tilt aftereffects for inner and outer tests (because these stimuli physically overlap with adapting stimulus sectors containing different orientations), and we should not observe a tilt aftereffect for central tests (because these stimuli overlap equally with both of the different orientations within adapting stimuli). These predictions accurately describe the pattern of results obtained during adapt-far, test-far runs of trials (inner, $t_{(7)}$ $=-3.55, p=0.009$; central, $t_{(7)}=-0.17, p=0.87$; outer, $t_{(7)}=$ $2.99, p=0.02$ ) (Fig. $2 d$ ). Of course, no size illusion should occur in these circumstances, because the adapting and test stimuli appeared to be located at the same distance from our observers.

A different situation should ensue during adapt-far, test-near runs of trials if the tuning of the tilt aftereffect is influenced by apparent size. Based on the results of the first experiment, during these conditions we would expect a $36 \%$ size illusion. This would shrink the apparent dimensions of test-near stimuli relative to the 
apparently more distant adapting stimulus. An illusion of this magnitude predicts that both inner and central test stimuli should now appear to correspond exclusively with the inner sectors of adapting stimuli. However, outer test stimuli should appear to correspond with regions associated with both the inner and outer sectors. Accordingly, we would predict tilt aftereffects in the same direction for inner and central test stimuli and that we should not observe a tilt aftereffect for outer test stimuli. Of course, these predictions should only hold if the appearance of stimuli overrides the physical dimensions of retinal input, which are unchanged relative to the adapt-far, test-far runs of trials.

Surprisingly, the predictions described above accurately describe the pattern of results obtained in adapt-far, test-near runs of trials (inner, $t_{(7)}=-7.30, p<0.001$; central, $t_{(7)}=-3.17, p=$ 0.016 ; outer, $t_{(7)}=0.85, p=0.426$ ) (Fig. $2 d$ ). These data show that, in these circumstances, the tuning of the tilt aftereffect can be predicted on the basis of apparent size, but not on the basis of the physical dimensions of retinal input.

During adapt-far, test-near runs of trials, the apparent dimensions of test stimuli were shrunk, relative to adapting stimuli, by making them appear relatively close to the observer. This situation can be reversed by instead making test stimuli appear further from the observer relative to adapting stimuli. Accordingly, we would predict an opposite pattern of results. We confirmed these predictions by completing adapt-near, test-far runs of trials (inner, $t_{(7)}=-2.18, p=0.066$; central, $t_{(7)}=2.582, p=0.036$; outer, $t_{(7)}=4.16, p=0.004$ ) (Fig. $2 d$ ). These additional runs of trials were completed by eight observers, six (including the authors) who had participated in the other experimental conditions and two who had not.

The strength of our manipulation is most apparent in the central test data (Fig. $2 d$ ). These test stimuli were physically identical across the different experimental conditions and overlapped equally with adapting stimulus regions tilted to the left and right of vertical (Fig. $2 c$ ). When these test stimuli appeared to be located in the same depth plane as the adapting stimuli (adapt-far, test-far), apparent test orientations were not influenced by adaptation. However, when these test stimuli appeared to be closer to the observer than the adapting stimuli (adapt-far, test-near), they appeared to be rotated away from the orientations within inner adaptor sectors. When they appeared to be further from the observer than adapting stimuli (adapt-near, test-far), they appeared rotated away from orientations within outer adaptor sectors. These data therefore reflect, not simply a modulation of tilt aftereffect strength, but a reversal in the contingency between adapting and test stimuli with illusory changes in size.

Note that in both the adapt-far, test-near and in the adaptnear, test-far conditions, observers shifted fixation between adapting and test stimulus presentations. However, the results obtained in these two conditions were markedly different. Thus, the shift in the position-based tuning of the tilt aftereffect cannot be attributed to the mere presence of fixation shifts. Instead, the results are marked by tuning shifts consistent with the magnitudes of illusory size changes predicted from experiment 1.

\section{Discussion}

Our data show that the position-based tuning of the tilt aftereffect is contingent on the perceived sizes of adapting and test stimuli, rather than simply being determined by the physical dimensions of retinal input. These effects were driven by manipulations of apparent viewing distance. Our data therefore provide the first human evidence for a shift in the tuning of a spatial coding interaction as a function of an illusory change in viewing distance.

Past physiological data have pointed to a possible interaction between viewing distance and spatial coding. For instance, the firing rates of neurons in monkey primary visual cortex have been shown to vary with viewing distance (Dobkins et al., 1998). Such changes at the level of single neurons are probably involved in implementing the spatial coding changes identified here.

An earlier psychophysical study found that the magnitude of spatial coding interactions can be modulated by gaze direction (Nishida et al., 2003). This finding complements our own in that both show that spatial coding interactions are dynamic and are therefore likely to be sensitive to the frequent gaze shifts made in daily life. Both observations considerably strengthen the probability that such interactions are functionally relevant in realworld settings (Barlow and Foldiak, 1989; Clifford et al., 2000; Dragoi et al., 2006).

Although our data show that the physical dimensions of retinal input are insufficient to explain the tuning of spatial coding interactions, we would not like to suggest that they are unimportant. The depth cues used here to induce a size illusion were consistent with a doubling of the viewing distance. Yet the magnitude of the size illusion was only $\sim 36 \%$, not the $100 \%$ predicted by the size-depth relationship. The tuning of spatial coding interactions was consistent with this smaller magnitude of illusion. Clearly, therefore, the depth cues provided were insufficient to completely overcome other constraining factors. One of these was probably the physical dimensions of retinal input.

The current data are consistent with some recent human brain imaging. These data showed that object images of the same physical dimensions could be associated with blood oxygenation level-dependent signals dispersed across different proportions of human primary visual cortex, depending on how far the objects appeared to be from the observer (Murray et al., 2006). Here we found that the position-based tuning of the tilt aftereffect is also dependent on apparent viewing distance. This last observation is interesting, because it has been shown that tilt aftereffect magnitude correlates with levels of adaptation in human primary visual cortex (Liu et al., 2007). In combination, the two datasets show that neither the spread of activity across the primary visual cortex (Murray et al., 2006) nor the position-based tuning of a spatial coding interaction that has been linked to this brain structure (Liu et al., 2007) is simply determined by the physical dimensions of retinal input. This convergence of evidence should guide and motivate further attempts to identify the neurophysiology underlying interactions between apparent viewing distance and spatial coding.

The precise functional mapping of adjacent cortical neurons onto adjacent retinal locations is one of the fundamental properties of visual brain structures (Daniel and Whitteridge, 1961; Sereno et al., 1995; Morgan, 2003). This arrangement is probably important for efficient spatial coding (Morgan, 2003). Datasets qualitatively similar to our own have been interpreted as suggesting that the mapping between cortical cells and the retinal surface/s is dynamic, such that a cortical cell might become responsive to a region on the retinal surface/s to which it was formerly unresponsive (Fu et al., 2004). We suggest an alternative. Apparent position shifts could instead be driven by intercortical and intracortical brain region interactions that modulate a cortical cell's response, without changing the locus on the retinal surface/s from which input is derived (Liu et al., 2006; Arnold et al., 2007; Viswanathan and Freeman, 2007). In the present context, such modulations could be driven by scaling processes driven by 
higher-level visual brain structures (Taira et al., 2000; Tsutsui et al., 2002; Sterzer and Rees, 2006). The present data cannot distinguish between these two possibilities [but see Arnold et al. (2007) for data concerning illusory motion-induced position shifts]. Additional work will therefore be required to discover the neurophysiological mechanism/s underlying the interactions between spatial coding and apparent viewing distance that have been identified here.

\section{References}

Arnold DH, Thompson M, Johnston A (2007) Motion and position coding. Vis Res 47:2403-2410.

Barlow HB, Foldiak P (1989) Adaptation and decorrelation in the cortex. In: The computing neuron (Durbin R, Miall C, Mitchison GJ, eds), pp 54-72. Wokingham, UK: Addison-Wesley.

Blakemore C, Carpenter RH, Georgeson MA (1970) Lateral inhibition between orientation detectors in the human visual system. Nature 228:37-39.

Brainard DH (1997) The psychophysics toolbox. Spat Vis 10:433-436.

Clifford CWG, Wenderoth P, Spehar B (2000) A functional angle on some after-effects in cortical vision. Proc R Soc Lond B 267:1705-1710.

Coltheart M (1971) Visual feature-analyzers and after-effects of tilt and curvature. Psych Rev 78:114-121.

Daniel PM, Whitteridge D (1961) The representation of the visual field on the cerebral cortex in monkeys. J Physiol (Lond) 159:203.

De Valois RL, De Valois KK (1991) Vernier acuity with stationary moving Gabors. Vis Res 31:1619-1626.

Dobkins AC, Jeo RM, Fiser J, Allman JM (1998) Distance modulation of neural activity in the visual cortex. Science 257:1279-1281.

Dragoi V, Sharma J, Miller EK, Sur M (2006) Dynamics of neuronal sensitivity in visual cortex and local feature discrimination. Nat Neurosci $5: 883-891$

Emmert E (1881) Größenverhaltnisse der Nachbilder. Klin Mbl Augenheilk 19:443-450.

Fang F, Murray SO, Kersten D, He S (2005) Orientation tuned FMRI adaptation in human visual cortex. J Neurophysiol 94:4188-4195.

Fu Y, Shen Y, Hongfeng G, Dan Y (2004) Asymmetry in visual cortical circuits underlying motion-induced perceptual mislocalization. J Neurosci 24:2165-2171.

Gibson JJ, Radner M (1947) Adaptation, after-effect, and contrast in the perception of tilted lines. I. Quantitative studies. J Exp Psychol 20:453-467.
Holway AH, Boring EG (1941) Determinants of apparent visual size with distance variant. Am J Psychol 54:21-37.

Joynson RB (1949) The problem of size and distance. Q J Exp Psychol 1:119-135.

Liu JV, Ashida H, Smith AT, Wandell BA (2006) Assessment of stimulusinduced changes in human V1 visual field maps. J Neurophysiol 96:3398-3408

Liu T, Larsson J, Carrasco M (2007) Feature-based attention modulates orientation-selective responses in human visual cortex. Neuron 55:313-323.

Morgan M (2003) The space between our ears. London: Weidenfeld and Nicolson.

Murray SO, Boyaci H, Kersten D (2006) The representation of perceived angular size in human primary visual cortex. Nat Neurosci 9:429-434.

Nishida S, Motoyoshi I, Andersen RA, Shimojo S (2003) Gaze modulation of visual aftereffects. Vis Res 43:639-649.

Ono H (1966) Distal and approximate size under reduced and non-reduced viewing conditions. Am J Psychol 79:234-241.

Pelli DG (1997) The VideoToolbox software for visual psychophysics: transforming numbers into movies. Spat Vis 10:437-442.

Sereno MI, Dale Am Reppas JB, Kwong KK, Belliveau JW, Brady TJ, Rosen BR, Tootell RB (1995) Borders of multiple visual areas in humans revealed by functional magnetic resonance imaging. Science 268:889-893.

Smith III EL, Chino YM, Ridder III WH, Kitagawa K, Langston A (1990) Orientation bias of neurons in the lateral geniculate nucleus of macaque monkeys. Vis Neurosci 5:525-545.

Sterzer P, Rees G (2006) Perceived size matters. Nat Neurosci 9:303-304.

Taira M, Tsutsui KI, Jiang M, Yara K, Sakata H (2000) Parietal neurons represent surface orientation from the gradient of binocular disparity. J Neurophysiol 83:3140-3146.

Tsutsui K, Sakata H, Naganuma T, Taira M (2002) Neural correlates for perception of 3D surface orientation from texture gradient. Science 298:409-412.

Viswanathan A, Freeman R (2007) Neurometabolic coupling in cerebral cortex reflects synaptic more than spiking activity Nat Neurosci 10:1308-1312.

Whitney D, Goltz HC, Thomas CG, Gati JS, Menon RS, Goodale MA (2003) Flexible retinotopy: motion-dependent position coding in the visual cortex. Science 302:878-881.

Xu X, Ichida J, Shostak Y, Bonds AB, Casagrande VA (2002) Are primate lateral geniculate nucleus (LGN) cells really sensitive to orientation or direction? Vis Neurosci 19:97-108. 